\title{
Stochastic Feedback and the Regulation of Biological Rhythms
}

\author{
Plamen Ch. Ivanov ${ }^{1}$, Luís A. Nunes Amaral ${ }^{1,2}$, \\ Ary L. Goldberger ${ }^{3}$, and H. Eugene Stanley ${ }^{1}$ \\ ${ }^{1}$ Center for Polymer Studies and Department of Physics, Boston University, Boston, MA 02215 \\ ${ }^{2}$ Condensed Matter Theory, Physics Department, Massachusetts Institute of Technology, \\ Cambridge, MA 02139 \\ ${ }^{3}$ Cardiovascular Division, Harvard Medical School, Beth Israel Hospital, Boston, MA 02215
}

\begin{abstract}
We propose a general approach to the question of how biological rhythms spontaneously self-regulate, based on the concept of "stochastic feedback". We illustrate this approach by considering the neuroautonomic regulation of the heart rate. The model generates complex dynamics and successfully accounts for key characteristics of cardiac variability, including the $1 / f$ power spectrum, the functional form and scaling of the distribution of variations, and correlations in the Fourier phases. Our results suggest that in healthy systems the control mechanisms operate to drive the system away from extreme values while not allowing it to settle down to a constant output.
\end{abstract}

PACS numbers: 02.50.Ey, 05.40, 05.45

Typeset using REVTEX 
The principle of homeostasis asserts that biological systems seek to maintain a constant output after perturbation [1]. Recent evidence, however, indicates that healthy systems even at rest display highly irregular dynamics [2]. Here, we address the paradox of how to reconcile homeostatic control and complex variability. The concept of dynamic equilibrium or homeostasis [1] led to the proposal that physiological variables, such as the cardiac interbeat interval $\tau(n)$, maintain an approximately constant value in spite of continual perturbations. Thus one can write in general

$$
\tau(n)=\tau_{0}+\eta,
$$

where $\tau_{0}$ is the "preferred level" for the interbeat interval and $\eta$ is a white noise with strength $\sigma$, defined as the standard deviation of $\eta$.

We first re-state this problem in the language of random walks. The time evolution of an uncorrelated and unbiased random walk is expressed by the equation $\tau(n+1)-\tau(n)=\eta$. The deviation from the initial level increases as $n^{1 / 2}$, so an uncorrelated and unbiased random walk does not preserve homeostasis (Fig. [17). To maintain a constant level, there must be a bias in the random walk [3],

$$
\tau(n+1)-\tau(n)=I(n),
$$

with

$$
I(n)= \begin{cases}w(1+\eta), & \text { if } \tau(n)<\tau_{0}, \\ -w(1+\eta), & \text { if } \tau(n) \geq \tau_{0} .\end{cases}
$$

The weight $w$ is the strength of the feedback input biasing the walker to return to its preferred level $\tau_{0}$.

We find that Eq. (2) does not reproduce the statistical properties of the empirical data (Fig. (1) $)$. We therefore generalize Eq. (2) to include several inputs $I_{k}(k=0,1, \cdots, m)$, with different preferred levels $\tau_{k}$, which compete in biasing the walker, and Eq. (2a) becomes

$$
\tau(n+1)-\tau(n)=\sum_{k=0}^{m} I_{k}(n),
$$


where

$$
I_{k}(n)= \begin{cases}w_{k}(1+\eta), & \text { if } \tau(n)<\tau_{k} \\ -w_{k}(1+\eta), & \text { if } \tau(n) \geq \tau_{k}\end{cases}
$$

From a biological point of view, it is clear that the preferred levels $\tau_{k}$ of the inputs $I_{k}$ cannot remain constant in time, for otherwise the system would not be able to respond to varying external stimuli. We assume that each preferred interval $\tau_{k}$ is a random function of time, with values correlated over a time scale $T_{\text {lock}}^{k}$. We next coarse grain the system and choose $\tau_{k}(n)$ to be a random step-like function constrained to have values within a certain interval and with the length of the steps drawn from a distribution with an average value $T_{\text {lock }}$ (Fig. 11c). This model yields several interesting features not fully explained by traditional models: (1) $1 / f$ power spectrum, (2) stable scaling form for the distribution of the variations in the beat-to-beat intervals and (3) Fourier phase correlations [四].

To illustrate the approach for the specific example of neuroautonomic control of cardiac dynamics, we first note that the healthy heart rate is determined by three major inputs: (i) the sinoatrial (SA) node; (ii) the parasympathetic (PS); and (iii) the sympathetic (SS) branches of the autonomic nervous system.

(i) The SA node or pacemaker is responsible for the initiation of each heart beat; in the absence of other external stimuli, it is able to maintain a constant interbeat interval [1]. Experiments in which PS and SS inputs are blocked reveal that the interbeat intervals are very regular and average only $0.6 \mathrm{~s}$ [5]. The input from the SA node, $I_{S A}$, thus biases the interbeat interval $\tau$ toward its intrinsic level $\tau_{S A}$ (see Fig. 11b).

(ii) The PS fibers conduct impulses that slow the heart rate. Suppression of SS stimuli, while under PS regulation, can result in the increase of the interbeat interval to as much as $1.5 \mathrm{~s}$ [5]. The activity of the PS system changes with external stimuli. We model these features of the PS input, $I_{P S}$, by the following conditions: (1) a preferred interval, $\tau_{P S}(n)$, randomly chosen from an uniform distribution with an average value larger than $\tau_{S A}$, and (2) a correlation time, $T_{P S}$, during which $\tau_{P S}$ does not change, where $T_{P S}$ is drawn from a 
distribution with an average value $T_{\text {lock }}$.

(iii) The SS fibers conduct impulses that speed up the heart beat. Abolition of parasympathetic influences when the sympathetic system remains active can decrease the interbeat intervals to less than $0.3 \mathrm{~s}$ [5]. There are several centers of sympathetic activity highly sensitive to environmental influences [5]. We represent each of the $N$ sympathetic inputs by $I_{S S}^{j}$ $(j=1, \cdots, N)$. We attribute to $I_{S S}^{j}$ the following characteristics: (1) a preferred interbeat interval $\tau_{S S}^{j}(n)$ randomly chosen from a uniform distribution with an average value smaller than $\tau_{S A}$, and (2) a correlation time $T_{j}$ in which $\tau_{S S}^{j}(n)$ does not change; $T_{j}$ is drawn from a distribution with an average value $T_{\text {lock }}$ which is the same for all $N$ inputs (and the same as for the PS system), so $T_{\text {lock }}$ is the characteristic time scale of both the PS and SS inputs.

The characteristics for the PS and SS inputs correspond to a random walk with stochastic feedback control (Fig. 11c). Thus, for the present example of cardiac neuroautonomic control, we have $N+2$ inputs and Eq. (3a) becomes

$$
\tau(n+1)-\tau(n)=I_{S A}(n)+I_{P S}\left(n, \tau_{P S}(n)\right)+\sum_{j=1}^{N} I_{S S}^{j}\left(n, \tau_{S S}^{j}(n)\right)
$$

where the structure of each input is identical to the one in Eq. (35). Equation (4) cannot fully reflect the complexity of the human cardiac system. However, it provides a general framework that can easily be extended to include other physiological systems (such as breathing, baroreflex control, different locking times for the inputs of the SS and PS systems [2,5], etc.). Equation (4) captures the essential ingredients responsible for a number of important statistical properties of the healthy heart rate.

To qualitatively test the model, we first compare the time series generated by the stochastic feedback model and the healthy heart and find that both signals display complex variability and patchiness (Fig. 2a). To quantitatively test the model, we compare the statistical properties of heart data with the predictions of the model:

(a) We first test for long-range anti-correlations in the interbeat intervals, which exist for healthy heart dynamics [6]. These anti-correlations can be uncovered by calculating power spectra, and we see (Fig. 2) that the model correctly reproduces the observed long-range 
anti-correlations. In particular, we note that the non-stationarity of both the data and model signals leads to the existence of several distinct scaling regimes in the power spectrum of $\tau(n)$ (Figs. 2 and 3). The stochastic feedback mechanism thus enables us to explain the formation of regions (patches) in the time series with different characteristics (see caption to Fig. (3).

(b) By studying the power spectrum of the increments we are able to circumvent the effects of the non-stationarity. Our results show that $1 / f$-scaling is indeed observed for the power spectrum of the increments, both for the data and for the model (Fig. 2).

(c) We calculate the probability density $P(A)$ of the amplitudes $A$ of the variations of interbeat intervals through the wavelet transform. It has been shown that the analysis of sequences of interbeat intervals with the wavelet transform [7] can reveal important scaling properties [8] for the distributions of the variations in complex nonstationary signals. In agreement with the results of Ref. [9], we find that the distribution $P(A)$ of the amplitudes $A$ of interbeat interval variations for the model decays exponentially - as is observed for

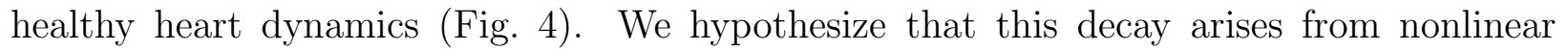
Fourier phase interactions and is related to the underlying nonlinear dynamics. To test this hypothesis, we perform a parallel analysis on a surrogate time series obtained by preserving the power spectrum but randomizing the Fourier phases of a signal generated by the model

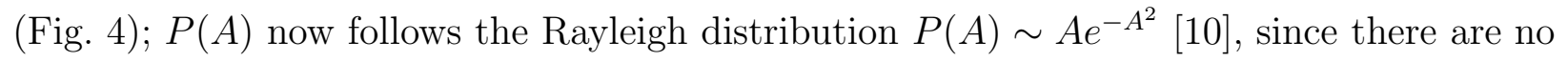
Fourier phase correlations.

(d) For the distribution displayed in Fig. 4, we test the stability of the scaling form at different time scales; we find that $P(A)$ for the model displays a scaling form stable over a range of time scales identical to the range for the data (Fig. 1) [9]. Such time scale invariance indicates statistical self-similarity [11].

A notable feature of the present model is that in addition to the power spectra, it accounts for the functional form and scaling properties of $P(A)$, which as we show are independent from the power spectra.

The model has a number of parameters, whose values may vary from one individual to 
another, so we next study the sensitivity of our results to variations in these parameters. We find that the model is robust to parameter changes. The value of $T_{\text {lock }}$ and the strength of the noise $\sigma$ are crucial to generate dynamics with scaling properties similar to those found for empirical data. We find that the model reproduces key features of the healthy heart dynamics for a wide range of time scales $\left(500 \leq T_{\text {lock }} \leq 2000\right)$ and noise strengths $(0.4 \leq \sigma \leq 0.6)$. The model is consistent with the existence of an extended "zone" in parameter space where scaling behavior holds, and our picture is supported by the variability in the parameters for healthy individuals for which similar scaling properties are observed.

The model, and the data which it fits, support a revised view of homeostasis that takes into account the fact that healthy systems under basal conditions, while being continuously driven away from extreme values, do not settle down to a constant output. Rather, a more realistic picture may involve nonlinear stochastic feedback mechanisms driving the system. 


\section{REFERENCES}

[1] C. Bernard, Les Phénoménes de la Vie (Paris, 1878); W. B. Cannon, Physiol. Rev. 9, 399 (1929).

[2] M. F. Shlesinger and B. J. West, in Random Fluctuations and Pattern Growth (Kluwer, Dordrecht, 1988).

[3] G. H. Weiss, Aspects and Applications of the Random Walk (Elsevier Science B.V., North-Holland, New-York, 1994).

[4] M. G. Rosenblum and J. Kurths, Physica A 215, 439 (1995); H. Seidel and H. P. Herzel, in Modelling the Dynamics of Biological Systems E. Mosekilde and O. G. Mouritsen, Eds. (Springer-Verlag, Berlin, 1995); J. M. Hausdorff and C. -K. Peng, Phys. Rev. E 54, 2154 (1996).

[5] R. M. Berne and M. N. Levy, Cardiovascular Physiology 6th ed. (C.V. Mosby, St. Louis, 1996).

[6] C. -K. Peng et al., Phys. Rev. Lett. 70, 1343 (1993).

[7] I. Daubechies, Comm. Pure and Appl. Math. 41, 909 (1988).

[8] J. F. Muzy, E. Bacry, and A. Arneodo, Int. J. Bifurc. Chaos 4, 245 (1994); A. Arneodo et al., Physica D 96, 291 (1996).

[9] P. Ch. Ivanov et al., Nature 383, 323 (1996).

[10] R. L. Stratonovich, Topics in the Theory of Random Noise (Gordon and Breach, New York, 1981).

[11] J. B. Bassingthwaighte, L. S. Liebovitch, and B. J. West, Fractal Physiology (Oxford Univ. Press, New York, 1994). 


\section{FIGURES}

a)

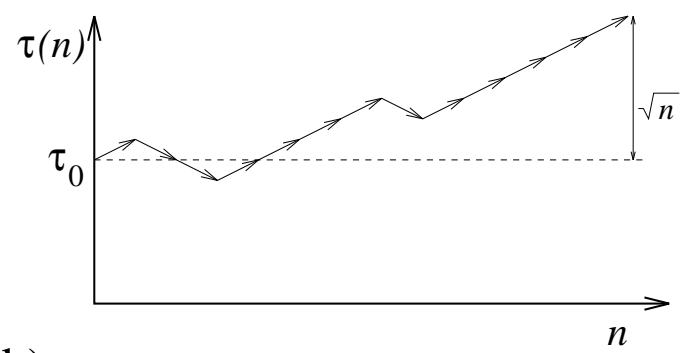

b)
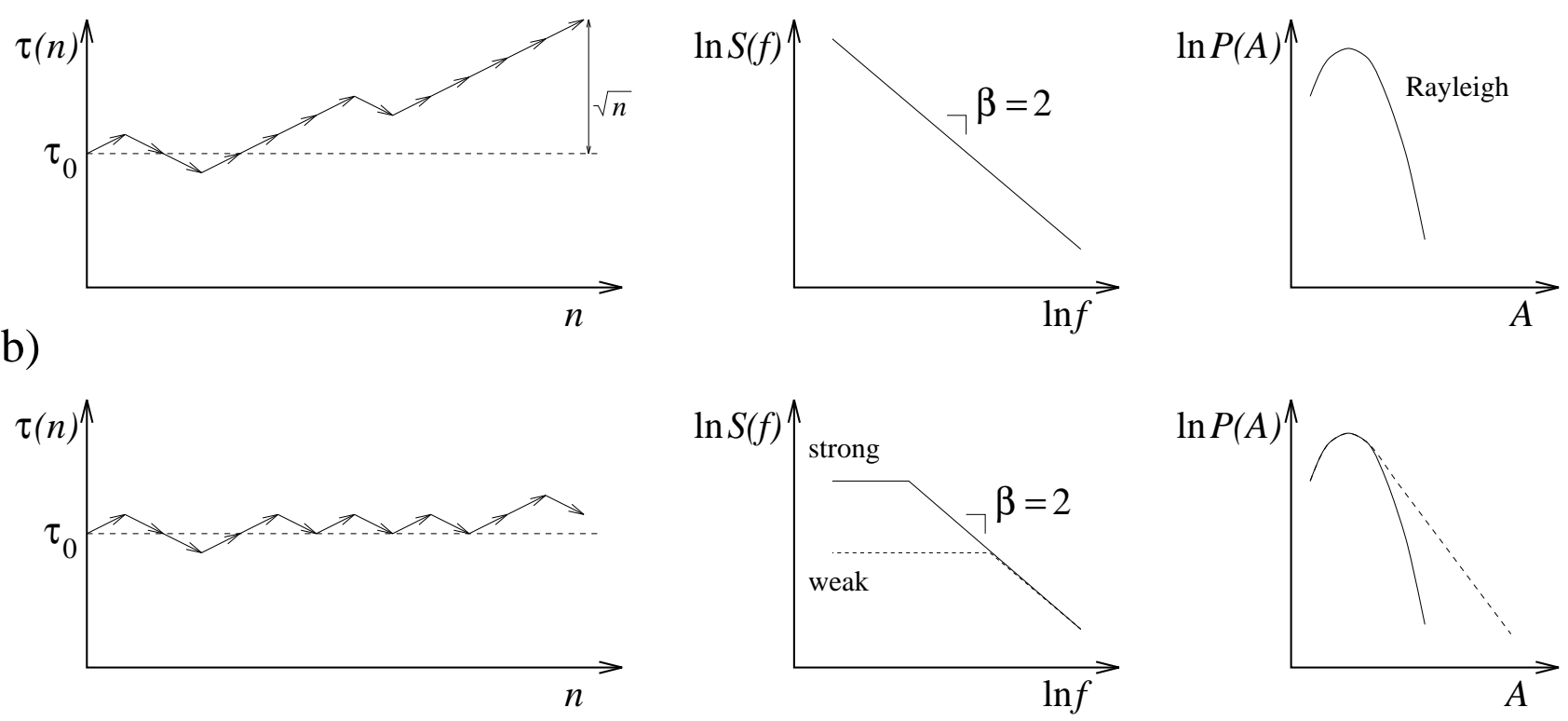

c)
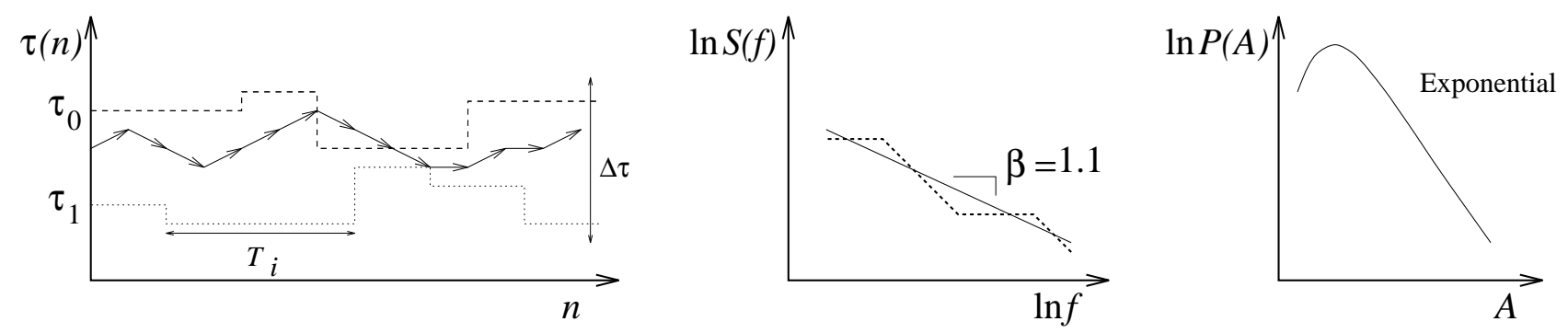
FIG. 1. Schematic representation of the dynamics of the model. (a) Evolution of a random walk starting from initial position $\tau_{0}$. The deviation of the walk from level $\tau_{0}$ increases as $n^{1 / 2}$, where $n$ is the number of steps. The power spectrum of the random walk scales as $1 / f^{2}$ (Brownian noise). The distribution $P(A)$ of the amplitudes $A$ of the variations in the interbeat intervals follows a Rayleigh distribution. (b) Random walk with a bias toward $\tau_{0}$. For short time scales (high frequencies), the power spectrum scales as $1 / f^{2}$ (Brownian noise) with a crossover to white noise at longer time scales due to the attraction to level $\tau_{0}$. Note the shift of the crossover to longer time scales (lower frequencies) when stronger noise is present. However, in both cases, $P(A)$ follows the Rayleigh distribution. (c) Random walk with two stochastic feedback controls. In contrast to (b), the levels of attraction $\tau_{0}$ and $\tau_{1}$ change values in time. Each level persists for a time interval $T_{i}$ drawn from a distribution with an average value $T_{\text {lock}}$. Perturbed by changing external stimuli, the system nevertheless remains within the bounds defined by $\Delta \tau$ even after many steps. We find that such dynamical mechanism, based on a single characteristic time scale $T_{\text {lock}}$, generates a $1 / f$ power spectrum over several decades. Moreover, $P(A)$ decays exponentially, which we attribute to nonlinear Fourier phase interactions in the walk. 

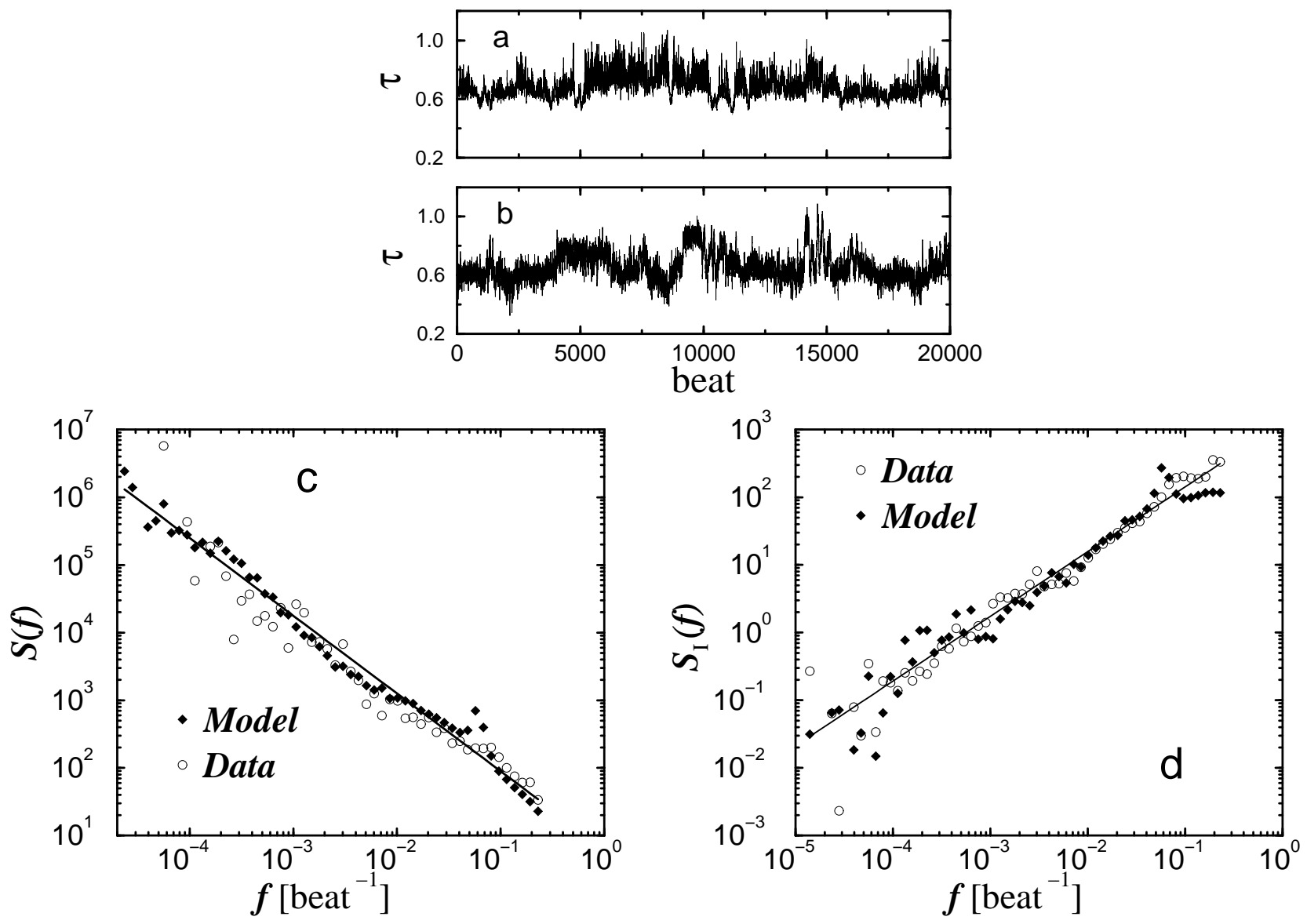
FIG. 2. (a) Sequence of interbeat intervals $\tau$ for a healthy individual. (b) Sequence of interbeat intervals for the model with parameters $N=7, w_{S A}=w_{S S}=w_{P S} / 3=0.01$ sec. For the results presented here, $T_{i}$ is randomly chosen from an exponential distribution with average $T_{\text {lock }}=1000$ beats, and $\eta$ is a symmetrical exponential distribution with zero average and standard deviation $\sigma=0.5$. The preferred values of the interbeat intervals for the different inputs were picked according to the following rules: (1) $\tau_{S A}=0.6 \mathrm{sec},(2) \tau_{P S}$ are randomly selected from an uniform distribution in the interval $[0.9,1.5] \mathrm{sec}$, and $(3)$ the $\tau_{S S}^{j}$ 's are randomly selected from an uniform distribution in the interval $[0.2,1.0] \mathrm{sec}$. We note that the actual value of the preferred interbeat intervals of the different inputs and the ratio between their weights are physiologically justified and are of no significance for the dynamics - they just set the range for the fluctuations of $\tau$, chosen to correspond to the empirical data. Also, any change of the shape of the distribution of the noise term or of the locking times leaves the reported here statistical properties of the generated signal unchanged. (c) Power spectra of the interbeat intervals $\tau(n)$ from the data and the model described by the relation $S(f) \sim 1 / f^{1.1}$. The presence of patches in both heart and model signals lead to observable crossovers embedded on this $1 / f$ behavior at different time scales. The local exponent $\beta$ from the power spectrum of $24 \mathrm{~h}$ records $\left(\approx 10^{5}\right.$ beats $)$ for 20 healthy subjects shows a persistent drift, so no true scaling exists. (d) Power spectra of the increments in $\tau(n)$. The model and the data both scale as power laws with exponents close to one. Since the non-stationarity is reduced, crossovers are no longer present. Here the local exponent $\beta_{I}$ fluctuates around an average value close to one, so true scaling does exist. 

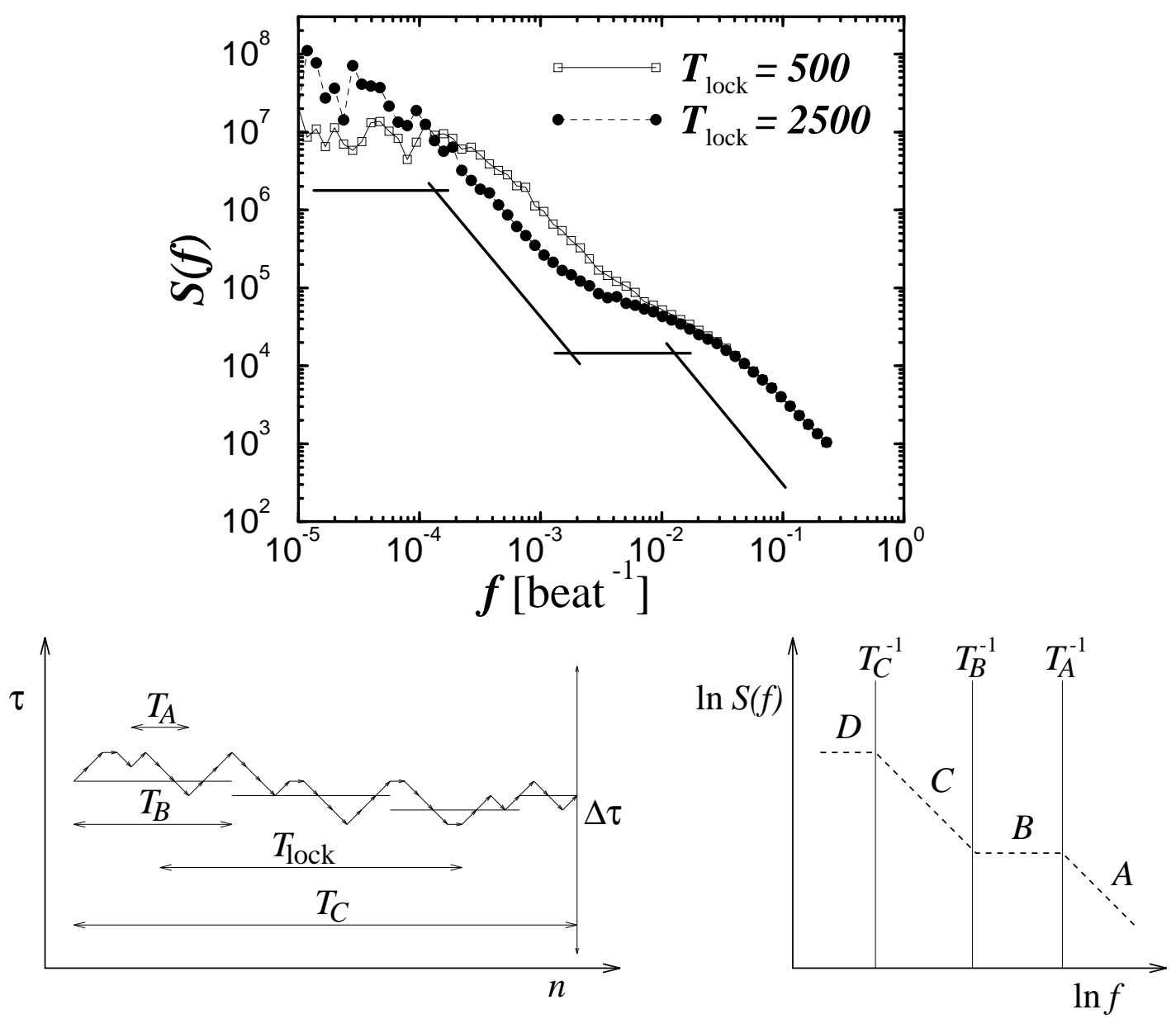
FIG. 3. Top: Effect of the correlation time $T_{\text {lock }}$ on the scaling of the power spectrum of $\tau(n)$ for a generated signal comprising $10^{6}$ beats. With increasing $T_{\text {lock }}$, the power spectrum does not follow a single power law but actually crosses over from a behavior of the type $1 / f^{2}$ at very small time scales (or high frequencies), to a behavior of the type $1 / f^{0}$ for intermediate time scales, followed by a new regime with $1 / f^{2}$ for larger time scales. At very large time scales, another regime appears with flat power spectrum. Bottom: Schematic diagram illustrating the origin of the different scaling regimes in the power spectrum of $\tau(n)$. For very short time scales, the noise will dominate, leading to a simple random walk behavior and $1 / f^{2}$ scaling (Region A). For time scales longer than $T_{A}$, the attraction towards the "average preferred level" of all inputs will dominate, leading to a flat power spectrum (Region B, see also Fig. 1 $1 \mathrm{~b}$ ). However, after a time $T_{B}$ (of the order of $\left.T_{\text {lock }} / N\right)$, the preferred level of one of the inputs will have changed, leading to the random drift of the average preferred level and the consequent drift of the walker towards it. So, at these time scales, the system can again be described as a simple random walker and we expect a power spectrum of the type $1 / f^{2}$ (Region C). Finally, for time scales larger than $T_{C}$, the walker will start to feel the presence of the bounds on the fluctuations of the preferred levels of the inputs. Thus, the power spectrum will again become flat (Region D). 

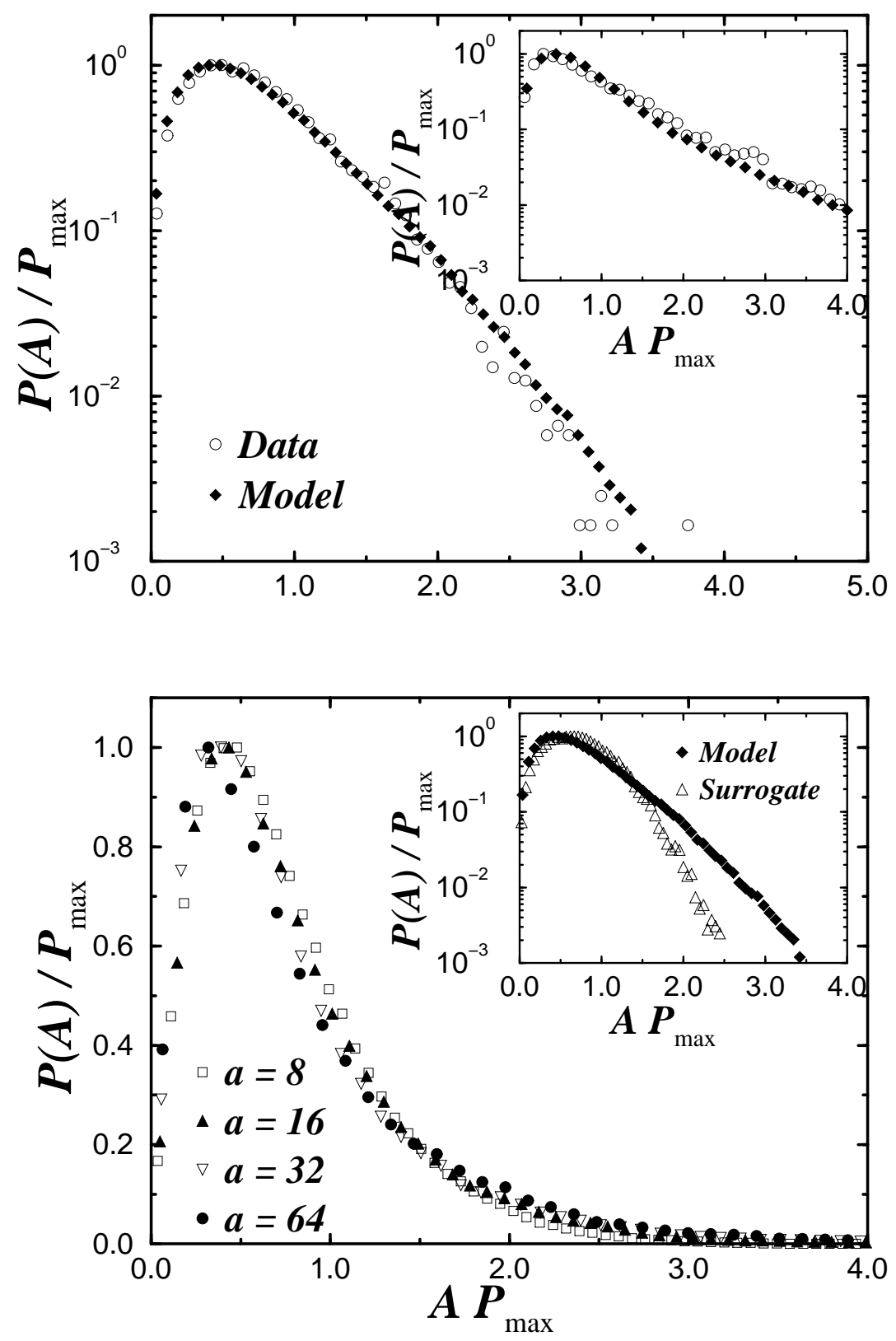
FIG. 4. We apply to the signal generated by the model the wavelet transform with fixed scale $a$, then use the Hilbert transform to calculate the amplitude $A$. Top: normalized histogram $P(A)$ for the data (6h daytime) and for the model (with the same parameter values as in Fig. 2), and for $a=8$ beats $(\approx 40 s)$. The inset shows a similar plot for data collected during the night and for the model with $N<w_{P S} / w_{S S}$. Note that the distribution is broader for this case with large values for the amplitudes deviating from the exponential tail. Bottom: we test the stability of the analysis for the model at different time scales $a$. The distribution is stable over a wide range of scales (identical to the range observed for heart data) and indicates statistical self-similarity in the variations at different time scales. We test the generated signal for nonlinearity and Fourier phase correlations, creating a surrogate signal by randomizing the Fourier phases of the generated signal but preserving the power spectrum (thus, leaving the results of Fig. 2 unchanged). The histogram of the amplitudes of variations for the surrogate signal follows the Rayleigh distribution, as expected theoretically (see inset). Thus the observed distribution which is universal for healthy cardiac dynamics, and reproduced by the model, reflects the Fourier phase interactions. 